\title{
INSERTION OF A MEASURABLE FUNCTION
}

\author{
WESLEY KOTZÉ and TOMASZ KUBIAK
}

(Received 4 March 1991; revised 3 July 1991)

Communicated by J. H. Rubinstein

\begin{abstract}
Some theorems on the existence of continuous real-valued functions on a topological space (for example, insertion, extension, and separation theorems) can be proved without involving uncountable unions of open sets. In particular, it is shown that well-known characterizations of normality (for example the Katětov-Tong insertion theorem, the Tietze extension theorem, Urysohn's lemma) are characterizations of normal $\sigma$-rings. Likewise, similar theorems about extremally disconnected spaces are true for $\sigma$-rings of a certain type. This $\sigma$-ring approach leads to general results on the existence of functions of class $\alpha$.

1991 Mathematics subject classification (Amer. Math. Soc.): 54C50, 28A05, 28A20, 54C20, 54C45, 26A21, 54C30.

Keywords and phrases: real-valued function, $\sigma$-ring, measurable function, class $\alpha$ function, insertion, extension, separation, perfect space, normal, extremally disconnected.
\end{abstract}

\section{Introduction}

In this paper we shall show that some important theorems on the existence of realvalued functions on a topological space remain valid if uncountable unions of open sets are not available. For instance, the proof of Urysohn's lemma depends upon constructing a countable family of open sets $\left\{U_{r}: r \in \mathbb{Q}\right\}$ such that $\bar{U}_{r} \subset U_{s}$ if $r<s$. This involves the operation of closure, hence a not necessarily countable intersection. But one can easily overcome this topological ingredient by requiring the existence of a countable family of closed sets $\{K: r \in \mathbb{Q}\}$ such that $U_{r} \subset K_{r} \subset U_{s}$ whenever $r<s$. In fact, an intervention of this type has already been done by Speed [15] who thus proved that a normal topology on a set can be replaced by a normal $\sigma$-ring.

We shall show that the other well-known characterizations of normality (that is, the Katêtov-Tong insertion theorem $[4,16]$ and the Tietze extension theorem) are characterizations of normal $\sigma$-rings. In fact, we shall do that for their abstract versions:

(C) 1994 Australian Mathematical Society 0263-61 15/94 \$A2.00+0.00 
the insertion theorem of Blair [1] and Lane [10], the extension theorem of Mrówka [11], and the Urysohn extension theorem of Gillman and Jerison [3]. Also, similar theorems about extremally disconnected spaces are shown to be true for sets equipped with extremally disconnected $\sigma$-rings (that is, $\sigma$-rings in which each two disjoint members are contained in disjoint complements of members of the ring).

However, the most important advantage of those observations is that one can substitute for a $\sigma$-ring the sets of additive class $\alpha$ in a perfect space and obtain corresponding theorems about real-valued functions of class $\alpha$ on a perfect space. In fact, those theorems (insertion, extension, and separation theorems) are characterizations of a wider class of spaces which will be called $\mathscr{A}_{\alpha}$-normal (the case $\alpha=1$ was discussed in [5] under the name $\mathrm{F}_{\sigma}$-normal spaces, where a Urysohn type lemma and a Tietze type theorem have been obtained directly). Another corollary is a classical theorem about insertion of a real-valued function of class $\alpha$ between two comparable upper and lower measurable functions of class $\alpha$ [14].

We note that the question of when a real-valued function other than a (uniformly [13]) continuous one (for example $[1,4,9,10,16]$ ) can be inserted between two comparable real-valued functions has rarely been investigated, especially for functions of higher classes with the notable exception of [5]. Results of similar type for class one functions of a real variable can be found, for instance, in [12].

This work was done while the second author was visiting Rhodes University as an F. R. D. and Hugh Kelly Research Fellow. Support from both these institutions is acknowledged with appreciation, also by the first author.

\section{Notation and terminology}

Our reference on rings of subsets and measurable functions is Sikorski [14]. One good reference for generating real-valued functions by monotone families of sets is Kutateladze [8]. For easy reference we recall some basic concepts and a few relevant facts about generating real-valued functions.

A subfamily $\mathscr{A} \subset \mathscr{P}(X)$ (the power set of a set $X$ ) is a ring on $X$ if $\mathscr{A}$ is closed under finite unions and finite intersections, and contains $\emptyset$ and $X$. If $\mathscr{A}$ is a ring, so is $\mathscr{A}_{c}=\{X \backslash A: A \in \mathscr{A}\}$. A ring is a $\sigma$-ring if it is closed under countable unions. If $S \subset X$, then $\mathscr{A} \mid S=\{A \cap S: A \in \mathscr{A}\}$ is a $\sigma$-ring on $S$ whenever $\mathscr{A}$ is a $\sigma$-ring on $X$. A ring $\mathscr{A}$ is a $\delta$-ring if $\mathscr{A}_{c}$ is a $\sigma$-ring. For $\mathscr{A}$ a ring, $\mathscr{A}_{\sigma}$ (respectively $\mathscr{A}_{\delta}$ ) is the $\sigma$-ring (respectively $\delta$-ring) consisting of all countable unions (respectively intersections) of members of $\mathscr{A}$.

The extended reals with usual ordering are denoted by $\bar{R}$. All functions considered in this paper are real-valued, that is $\overrightarrow{\mathrm{R}}$ - or Re-valued (the latter are called finite functions). For $f$ a function on $X$ and $a \in \operatorname{Re},[f>a]=\{x \in X: f(x)>a\}$ and 
$[f<a]=\{x \in X: f(x)<a\}$. Also, $[f \geq a]=X \backslash[f<a]$, etcetera. We write $f \leq g$ if $f(x) \leq g(x)$ for all $x$ in $X$.

Let $\mathscr{A}$ be a $\sigma$-ring on $X$. A function $f$ on $X$ is called lower (respectively upper) $\mathscr{A}$-measurable if $[f>a] \in \mathscr{A}$ (respectively $[f<a] \in \mathscr{A}$ ) for each $a \in$ Re. We say that $f$ is $\mathscr{A}$-measurable if it is both lower and upper $\mathscr{A}$-measurable. We shall say that two subsets $A$ and $B$ of $X$ are completely $\mathscr{A}$-separated if there is an $\mathscr{A}$-measurable function $f$ on $X$ such that $A \subset[f \leq a]$ and $B \subset[f \geq b]$ for some $a<b$ in Re.

Each increasing family $\mathscr{F}=\left\{F_{r}: r \in \mathbb{Q}\right\} \subset \mathscr{P}(X)$ is called a scale on $X$. For future use we quote the following well-known facts.

PROPOSITION 2.1. Let $\mathscr{F}$ be a scale on $X$. Then the following hold.

(1) For each $x \in X, f(x)=\inf \left\{r \in \mathbb{Q}: x \in F_{r}\right\}$ defines an $\overrightarrow{\mathrm{R}}$-valued function on $X$. (We shall say $f$ is generated by $\mathscr{F}$.)

(2) If $f$ is generated by $\mathscr{F}$, then $[f<a]=\bigcup\left\{F_{r}: r<a\right\}$ and $[f \leq a]=\bigcap\left\{F_{r}\right.$ : $r>a\}$ for all $a \in \mathrm{Re}$.

(3) If $g$ and $h$ are generated by $\mathscr{G}$ and $\mathscr{H}$, respectively, then $g \leq h$ if and only if $H_{r} \subset G_{s}$ whenever $r<s$.

(4) If $f$ is generated by $\mathscr{F}$, then $f(x)>-\infty$ [respectively $<+\infty$ ] for all $x \in X$ if and only if $\bigcap \mathscr{F}=\emptyset[$ respectively $\bigcup \mathscr{F}=X]$.

(Therefore a scale $\mathscr{F}$ satisfying both $\bigcap \mathscr{F}=\emptyset$ and $\bigcup \mathscr{F}=X$ will be called a finite scale,)

\section{Insertion and extension of a measurable function}

We shall use the machinery developed by Katětov [4].

A binary relation $\rho$ on $\mathscr{P}(X)$ will be called a $K$-relation if it satisfies the following conditions:

(i) If $A_{i} \rho B_{j}(1 \leq i \leq m, 1 \leq j \leq n)$, then there exists $C \in \mathscr{P}(X)$ such that $A_{i} \rho C$ and $C \rho B_{j}$ for all those $i$ and $j$.

(ii) $A \rho B$ implies $A \subset B$.

(iii) $A \subset B$ implies $A \bar{\rho} B$, where $A \bar{\rho} B$ if and only if $B \rho C$ implies $A \rho C$, and $D \rho A$ implies $D \rho B$ for any $C, D \in \mathscr{P}(X)$

We note that (iii) is equivalent to the following statement:

(iv) If $C \subset A, A \rho B$, and $B \subset D$, then $C \rho D$ for every $A, B, C, D \in \mathscr{P}(X)$

LEMMA 3.1. (Katětov [4]). Let $\rho$ be a $K$-relation on $\mathscr{P}(X)$. Let $\mathscr{G}=\left\{G_{r}: r \in \mathbb{Q}\right\}$ and $\mathscr{H}=\left\{H_{r}: r \in \mathbb{Q}\right\}$ be such that $H_{r} \rho G_{s}, H_{r} \bar{\rho} H_{s}$, and $G_{r} \bar{\rho} G_{s}$ whenever $r<s$. Then there exists $\mathscr{F}=\left\{F_{r}: r \in \mathbb{Q}\right\}$ with $H_{r} \rho F_{s}, F_{r} \rho F_{s}$, and $F_{r} \rho G_{s}$ whenever $r<s$. 
REMARK 3.2. We shall deal only with the case when $\mathscr{G}$ and $\mathscr{H}$ are increasing (scales). Then the conditions $G_{r} \bar{\rho} G_{s}$ and $H_{r} \bar{\rho} H_{s}(r<s)$ hold trivially. Also, we shall only require $\mathscr{F}$ to satisfy $H_{r} \subset F_{s}, F_{r} \rho F_{s}$, and $F_{r} \subset G_{s}$ if $r<s$.

DEFINITION 3.3. Let $\mathscr{A}$ be a ring on $X$. An arbitrary relation $\rho$ on $\mathscr{P}(X)$ is called an $\mathscr{A}$-separating relation if for every $A, B \in \mathscr{P}(X), A \rho B$ implies there exist $C \in \mathscr{A}$ and $\mathscr{D} \in \mathscr{A}_{\mathrm{c}}$ such that $A \subset C \subset B$ and $A \subset D \subset B$.

Note that $\rho$ is $\mathscr{A}$-separating if and only if it is $\mathscr{A}_{c}$-separating.

EXAMPLE 3.4. (1) If $\mathscr{A}$ is a topology on $X$, then $A$ is in an $\mathscr{A}$-separating relation to $B$ if and only if $A$ and $X \backslash B$ are topologically separated (that is, $\bar{A} \subset B$ and $A \subset$ Int $B$ ).

(2) For $\mathscr{A}$ a $\sigma$-ring on $X$, the relation $\rho$ on $\mathscr{P}(X)$ defined by $A \rho B$ if and only if $A$ and $X \backslash B$ are completely $\mathscr{A}$-separated is an $\mathscr{A}$-separating $K$-relation. [Indeed, since $A \subset[f \leq 0] \subset[f<1] \subset B$ with $f \mathscr{A}$-measurable, hence (ii) and (iv) hold trivially. If $A_{i} \rho B_{j}$ and $f_{i j}(1 \leq i \leq m, 1 \leq j \leq n)$ are the corresponding $\mathscr{A}$-measurable functions, then $g=\inf _{i} \sup _{j} f_{i j}$ is $\mathscr{A}$-measurable and satisfies $A_{i} \subset[g \leq 0] \subset[g<$ 1] $\subset B_{j}$ for all $i$ and $j$. Therefore $A_{i} \rho C \rho B_{j}$ with $C=\left[g<\frac{1}{2}\right]$.]

LEMMA 3.5. If $\mathscr{A}$ is a $\sigma$-ring on $X, \mathscr{F}$ is a scale, and $\rho$ is an $\mathscr{A}$-separating relation on $\mathscr{P}(X)$ such that $F_{r} \rho F_{s}$ whenever $r<s$, then the function $f$ generated by $\mathscr{F}$ is $\mathscr{A}$-measurable.

PROOF. For $r<s$, let $C_{r s}$ and $D_{r s}$ be the corresponding members of $\mathscr{A}_{\text {and }} \mathscr{A}_{c}$, respectively, such that $F_{r} \subset C_{r s} \subset F_{s}$ and $F_{r} \subset D_{r s} \subset F_{s}$. Clearly, $\bigcup\left\{F_{r}: r<a\right\}=$ $\bigcup\left\{C_{r s}: r<s<a\right\} \in \mathscr{A}$ and $\bigcap\left\{F_{r}: r>a\right\}=\bigcap\left\{D_{r s}: s>r>a\right\} \in \mathscr{A}_{c}$ for all $a \in \operatorname{Re}$. By Proposition 2.1(2), $f$ is $\mathscr{A}$-measurable.

THEOREM 3.6. (Insertion Theorem). Let $\mathscr{A}$ be a $\sigma$-ring on $X$. Let $g \leq h$ be two $\overline{\mathrm{R}}$-valued functions on $X$. The following statements are equivalent

(1) There exists an $\mathscr{A}$-measurable $\overline{\mathrm{R}}$-valued function $f$ on $X$ such that $g \leq f \leq h$.

(2) For each $a<b$ in $\operatorname{Re},[h \leq a]$ and $[g \geq b]$ are completely $\mathscr{A}$-separated.

(3) There exist scales $\mathscr{G}$ and $\mathscr{H}$ generating $g$ and $h$, respectively such that $H_{r}$ and $X \backslash G_{s}$ are completely $\mathscr{A}$-separated whenever $r<s$.

(4) There exist scales $\mathscr{G}$ and $\mathscr{H}$ generating $g$ and $h$, respectively, and an $\mathscr{A}$ separating $K$-relation $\rho$ on $\mathscr{P}(X)$ such that $H_{r} \rho G_{s}$ if $r<s$.

ProOF. (1) implies (2) is trivial.

(2) implies (3): for each $r \in \mathbb{Q}$, put $G_{r}=[g<r]$ and $H_{r}=[h \leq r]$.

(3) implies (4): by Example 3.4(2). 
(4) implies (1): by Lemma 3.1 (see also Remark 3.2), there exists $\mathscr{F}=\left\{F_{r}: r \in \mathbb{Q}\right\}$ such that $F_{r} \rho F_{s}$ (hence $\mathscr{F}$ is a scale by (ii)), $H_{r} \subset F_{s}$, and $F_{r} \subset G_{s}$ if $r<s$. Thus, by Proposition 2.1(3), the function $f$ generated by $\mathscr{F}$ satisfies $g \leq f \leq h$. Since $\rho$ is $\mathscr{A}$-separating, $f$ is $\mathscr{A}$-measurable by Lemma 3.5.

REMARK 3.7. Of course, if $g$ and $h$ are finite functions, so is $f$. For this case and with $\mathscr{A}$ a topology on $X$ (then $\mathscr{A}$-measurability = continuity), the equivalence of (1) and (2) in Theorem 3.6 was proved by Blair [1, 3, 5], and (1) if and only if (2) if and only if (3) was proved independently by Lane [10, Theorem 2.1]; (4) if and only if (1) is in Lane [9, Theorem 1]. Note in passing that Lane $[9,10]$ uses the concept of lower indefinite cut sets: if $f$ is an Re-valued function on $X$ and $[f<a] \subset A(f, a) \subset[f \leq a]$ for all $a \in \operatorname{Re}$, then $A(f, a)$ is called a lower indefinite cut set of $f$ at $a$. Of course, $\{A(f, r): r \in \mathbb{Q}\}$ is a finite scale that generates $f$. Also, any other scale generating $f,\left\{F_{r}: r \in \mathbb{Q}\right\}$ say, must satisfy $[f<r] \subset F_{r} \subset[f \leq r]$ for all $r \in \mathbb{Q}$. Therefore, Lane's families of cut sets are actually finite scales.

Note also that Theorem 3.6 partially improves [13, Theorem 3.4], which in turn is an $\bar{R}$-valued version of the mentioned results of Blair [1] and Lane [10].

The following extension theorem for bounded functions is an immediate consequence of Theorem 3.6. We include a proof (whose technique is well-known) for completeness.

THEOREM 3.8. (Mrówka Extension Theorem). Let $\mathscr{A}$ be a $\sigma$-ring on $X$ and $S \subset X$. Let $f: S \rightarrow[0,1]$ be $(\mathscr{A} \mid S)$-measurable. Then the following are equivalent:

(1) $f$ has an $\mathscr{A}$-measurable extension over $X$.

(2) If $a<b$ in $[0,1]$, then $[f \leq b]$ and $[f \geq b]$ are completely $\mathscr{A}$-separated.

PROOF. (1) implies (2): let $g$ be an extension of $f$. Then $[f \leq a] \subset[g \leq a]$ and $[f \geq b] \subset[g \geq b]$, that is, $g$ separates these sets.

(2) implies (1): define $g, h: X \rightarrow[0,1]$ by $g=h=f$ on $S, g=0$ on $X \backslash S$, and $h=1$ on $X \backslash S$. Then $[g \geq b]=[f \geq b]$ if $b>0$, and $[h \leq a]=[f \leq a]$ if $a<1$. Therefore if $a<b$ in [0,1], then $[h \leq a]$ and [ $g \geq b]$ are completely $\mathscr{A}$-separated, that is, Statement 3.6(2) holds. Since $g \leq h$, by Statement 3.6(1) there exists an $\mathscr{A}$-measurable function $f^{*}$ on $X$ such that $g \leq f^{*} \leq h$, which is therefore the required extension of $f$.

COROLlaRY 3.9. (Urysohn Extension Theorem). For $\mathscr{A}$ a $\sigma$-ring on $X$ and $S \subset X$, the following statements are equivalent:

(1) Each $(\mathscr{A} \mid S)$-measurable function $f: S \rightarrow[0,1]$ has an $\mathscr{A}$-measurable extension over $X$. 
(2) Each two completely $(\mathscr{A} \mid S)$-separated (in S) subsets of $S$ are completely $\mathscr{A}$ separated in $X$.

PROOF. (1) implies (2): if $f$ completely $(\mathscr{A} \mid S)$-separates $A$ and $B$ in $S$, and if $g$ is an $\mathscr{A}$-measurable extension of $f$, then $A \subset[f=O] \subset[g=0]$ and $B \subset[f=1] \subset[g=1]$.

(2) implies (1): let $f: S \rightarrow[0,1]$ be $(\mathscr{A} \mid S)$-measurable. Then for each $a<b$ in $[0,1],[f \leq a]$ and $[f \geq b]$ are completely $(\mathscr{A} \mid S)$-separated (by $f$ ). Therefore, $f$ has a continuous extension to $X$ by Theorem 3.8 .

REMARK 3.10. With $\mathscr{A}$ a topology on $X$, Theorem 3.8 is due to Mrówka [11, 4.11], and Corollary 3.9 is known as the Urysohn Extension Theorem [3, 1.17].

\section{Perfect rings, normal rings, and extremally disconnected rings}

The concepts of normality and perfectness of a topology of a space can easily be extended to rings of subsets. Recall that a perfect space is one in which each open set is $\mathrm{F}_{\sigma}$.

A ring $\mathscr{A}$ on a set $X$ is normal if, given any two disjoint members of $\mathscr{A}_{c}$, there are disjoint members of $\mathscr{A}$ containing them respectively. A ring $\mathscr{A}$ is called perfect if $\mathscr{A} \subset \mathscr{A}_{c \sigma}$ (that is, if each member of $\mathscr{A}$ is a countable union of complements of $\mathscr{A})$; equivalently, if $\mathscr{A}_{c} \subset \mathscr{A}_{\delta}$. We shall say that a ring $\mathscr{A}$ is extremally disconnected if $\mathscr{A}_{c}$ is normal. (We note that in [15] a ring $\mathscr{A}$ is called normal if the ring $\mathscr{A}_{c}$ is normal in the sense above. Such a terminology, however, is inconsistent with extremal disconnectedness of general topology; see the example which follows.)

EXAMPLE 4.1. For $(X, \mathscr{A})$ a topological space, $X$ is normal [extremally disconnected] if and only if $\mathscr{A}$ is normal [extremally disconnected]. For further examples, see Remarks 4.7 and 5.2, and Proposition 5.3. (Recall that a space is extremally disconnected if and only if every open set has an open closure if and only if disjoint open sets have disjoint closures, and the latter is clearly equivalent to the statement that each two disjoint open sets are contained in disjoint closed sets.)

We now specialize Theorem 3.6 for the case when $\mathscr{A}$ is normal or extremally disconnected.

LEMMA 4.2. Let $\mathscr{A}$ be a normal ring on $X$. Then the relation $\rho$ on $\mathscr{P}(X)$, defined by $A \rho B$ if and only if there exist $C \in \mathscr{A}_{c}$ and $D \in \mathscr{A}$ with $A \subset C \subset D \subset B$, is an $\mathscr{A}$-separating $K$-relation. 
PROOF. Each $\mathscr{A}$-separating $\rho$ satisfies (ii), and (iv) holds trivially. So, we verify (i). For if $A_{i} \rho B_{j}$, and $C_{i j}, D_{i j}$ are the corresponding members of $\mathscr{A}_{c}$ and $\mathscr{A}$, respectively $(1 \leq i \leq m, 1 \leq j \leq n)$, then $C=\bigcup_{i=1}^{m} \bigcap_{j=1}^{n} C_{i j} \in \mathscr{A}_{c}, D=\bigcup_{i=1}^{m} \bigcap_{j=1}^{n} D_{i j} \in \mathscr{A}$, and $\bigcup_{i=1}^{m} A_{i} \subset C \subset D \subset \bigcap_{j=1}^{n} B_{j}$. Since $\mathscr{A}$ is normal there exist $D_{1} \in \mathscr{A}$ and $C_{1} \in \mathscr{A}_{c}$ such that $C \subset D_{1} \subset C_{1} \subset D$. Therefore $A_{i} \rho D_{1} \rho B_{j}$ for all those $i$ and $j$.

COROLLARY 4.3. Let $\mathscr{A}$ be an extremally disconnected ring on $X$. Then the relation $\rho$ on $\mathscr{P}(X)$, defined by $A \rho B$ if and only if there exist $C \in \mathscr{A}$ and $D \in \mathscr{A}_{c}$ with $A \subset C \subset D \subset B$, is an $\mathscr{A}$-separating $K$-relation.

PROOF. $\mathscr{A}_{c}$ is normal and $\mathscr{A}_{c c}=A$, so Lemma 4.2 applies.

Note that this sort of 'duality' between normality and extremal disconnectedness, discussed in [6], appears to be a very efficient source of deriving new results about normality from extremal disconnectedness and vice versa. The following provides examples of such dualities.

THEOREM 4.4. Let $\mathscr{A}$ be a $\sigma$-ring on $X$. Then the following statements are equivalent:

(1) A is normal (extremally disconnected).

(2) (Insertion) If $g \leq h$ [respectively $h \leq g$ ] are real-valued functions on $X$ such that $g$ is upper and $h$ is lower $\mathscr{A}$-measurable, then there exists an $\mathscr{A}$-measurable function $f$ on $X$ such that $g \leq f \leq h$ [respectively $g \leq f \leq h$ ].

(3) (Separation) Each two disjoint $A$ and $B$ in $\mathscr{A}_{c}$ [respectively $\mathscr{A}$ ] are completely $\mathscr{A}$-separated.

(4) (Extension) For every $S \in \mathscr{A}_{c}$ [respectively $\mathscr{A}$ ] each $(\mathscr{A} \mid S)$-measurable function $f: S \rightarrow[0,1]$ has an $\mathscr{A}$-measurable extension to all of $X$.

Proof. (1) implies (2): define $G_{r}=[g<r]$ and $H_{r}=[h \leq r]$ for all $r \in \mathbb{Q}$. Clearly, $\left\{G_{r}\right\}$ and $\left\{H_{r}\right\}$ are scales generating $g$ and $h$ (finite scales if $g$ and $h$ are finite functions), respectively. Also, $H_{r} \subset G_{s}$ (respectively $G_{r} \subset H_{s}$ ) if $r<s$. And since $H_{r} \in \mathscr{A}_{c}, G_{r} \in \mathscr{A}$ for all $r$, in fact, $H_{r} \rho G_{s}$ [respectively $G_{r} \rho H_{s}$ ] if $r<s$, where $\rho$ is the relation of Lemma 4.2 (respectively Corollary 4.3). Thus, by Theorem 3.6(4), there exists an $\mathscr{A}$-measurable function $f$ such that $g \leq f \leq h$ (respectively $h \leq f \leq g$ ).

(2) implies (3): if $A, B \in \mathscr{A}_{c}$ (respectively $\mathscr{A}$ ) are disjoint, then $\chi_{A} \leq \chi_{X \backslash B}, \chi_{A}$ is upper (respectively lower) and $\chi_{X \backslash B}$ is lower (respectively upper) $\mathscr{A}$-measurable. Then the $\mathscr{A}$-measurable function $f$ between $\chi_{A}$ and $\chi_{X \backslash B}$ has the required property.

(3) implies (4), for $\mathscr{A}_{c}$ : for any $a<b$ in $[0,1],[f \leq a]$ and $[f \geq b]$ are in $(\mathscr{A} \mid S)_{c}$. Since $S \in \mathscr{A}_{c}$ we have $(\mathscr{A} \mid S)_{c} \subset \mathscr{A}_{c}$. By Theorem 3.8, $f$ has an $\mathscr{A}$-measurable extension. 
(3) implies (4), for $\mathscr{A}$ : let $a<b$ in [0,1]. If $a<c<d<b$, then $C=[f<c]$ and $D=[f>d]$ are in $\mathscr{A} \mid S$ and $\mathscr{A} \mid S \subset \mathscr{A}$ since $S \in \mathscr{A}$. Thus $C$ and $D$ are completely $\mathscr{A}$-separated and so are $[f \leq a] \subset C$ and $[f \geq b] \subset D$. By Theorem 3.8, $f$ is extendable to an $\mathscr{A}$-measurable function.

(4) implies (1): for each two disjoint $A$ and $B$ in $\mathscr{A}_{c}$ (respectively $\mathscr{A}$ ), the function $f$ from $S=A \cup B$ to $[0,1]$, defined by and $f=0$ on $A$ and $f=1$ on $B$, is $(\mathscr{A} \mid S)$-measurable, hence extendable to an $\mathscr{A}$-measurable function $f^{*}$. Clearly, $A \subset\left[f^{*}<\frac{1}{2}\right]$ and $B \subset\left[f^{*}>\frac{1}{2}\right]$ (respectively $A \subset\left[f^{*} \leq \frac{1}{2}\right]$ and $B \subset\left[f^{*} \geq \frac{3}{4}\right]$ ), which proves the assertion.

REMARK 4.5. (1) First note that (4) above is easily obtainable from (2) by avoiding (3) and, thus, Theorem 3.8 as well. Indeed, $g$ and $h$ defined as in the proof of Theorem 3.8 are, respectively, upper and lower (respectively lower and upper) $\mathscr{A}$-measurable whenever $S \in \mathscr{A}_{c}$ (respectively $\mathscr{A}$ ). Therefore the $\mathscr{A}$-measurable function, which does exist by Theorem 4.4(2), is the required extension. In other words, one can derive extension from insertion by avoiding separation.

(2) If $\mathscr{A}$ is an extremally disconnected topology, then (1) if and only if (2) for finite functions is in Lane [9, Corollary 4], and (1) if and only if (3) if and only if (4) is in Gillman and Jerison $[3,1 . \mathrm{H}]$.

(3) That (1) if and only if (3) for $\mathscr{A}$ a normal $\sigma$-ring is proved by Speed [15, Theorem 3.1] (cf. 4.1) by modifying the usual Urysohn's procedure (see Section 1).

(4) Of course, if $\mathscr{A}$ is a normal topology, the statements (2), (3), and (4) become, respectively, the Katětov-Tong theorem [4, 16], Urysohn's lemma, and the Tietze extension theorem.

REMARK 4.6. Another area which is covered by the normality case of Theorem 4.4 are $F_{\sigma}$-normal spaces of [5] (this class of spaces includes all perfect spaces, that is wellbehaved spaces in the terminology of [5]): a topological space is called $F_{\sigma}$-normal if for each two disjoint $G_{\delta}$-sets there exist two disjoint $F_{\sigma}$-sets containing them. In other words, a space is $F_{\sigma}$-normal if and only if $\mathscr{F}_{\sigma}$, the $\sigma$-ring of all $F_{\sigma}$-sets, is normal. With $\mathscr{A}=\mathscr{F}_{\sigma}$, the normality cases of (1) if and only if (3) and (1) if and only if (4) of Theorem 4.4 become Theorem 2.3 and 2.10 of [5], respectively. Note that real valued $\mathscr{F}_{\sigma}$-measurable functions are class one functions. Those two results of [5] are of course simple consequences (compare Remark 4.5(1)) of the following insertion theorem (that is, (1) if and only if (2) of Theorem 4.4 with $\mathscr{A}=\mathscr{F}_{\sigma}$ ): $A$ topological space $X$ is $F_{\sigma}$-normal if and only if, whenever $g \leq h$ are upper and lower $\mathscr{F}_{\sigma}$-measurable functions on $X$ respectively, there exists a class one function $f$ on $X$ such that $g \leq f \leq h$. (In [5], upper and lower $\mathscr{F}_{\sigma}$-measurable functions are called upper semi- $K_{1}$ and lower semi- $K_{1}$, respectively.) These ideas can be performed for $\sigma$-rings of (higher than 1) additive class $\alpha$ in a topological space, at least in a perfect space. 


\section{Insertion and extension of functions of class $\alpha$}

As mentioned in Remark 4.6, each result of Section 4 goes over to measurable functions of class $\alpha$ on a perfect space; also see Remark 5.4. Therefore recall that the classification of Borel sets in a metric space is still valid in a perfect space (see $[2,1.7 .5])$. In fact, the same classification can be performed in a set equipped with a perfect $\sigma$-ring as actually done in [14, Chapter V, Section 9]:

Let $\mathscr{A}$ be a perfect $\sigma$-ring on a set $X$, let $\mathscr{A}_{0}=\mathscr{A}_{\text {and }} \mathscr{B}_{0}=\mathscr{A}_{c}$, and let for each countable ordinal $\alpha>0, \mathscr{A}_{\alpha}=\left(\bigcup_{\beta<\alpha} \mathscr{B}_{\beta}\right)_{\sigma}$ and $\mathscr{B}_{\alpha}=\left(\bigcup_{\beta<\alpha} \mathscr{A}_{\beta}\right)_{\delta}$. Then for each $\alpha>0$

(I) $\mathscr{A}_{\alpha}$ is a $\sigma$-ring and $\mathscr{B}_{\alpha}=\left(\mathscr{A}_{\alpha}\right)_{c}$ (hence a $\delta$-ring).

(II) If $0 \leq \beta<\alpha$, then $\mathscr{A}_{\beta} \cup \mathscr{B}_{\beta} \subset \mathscr{A}_{\alpha} \cap \mathscr{B}_{\alpha}$.

Note that the above construction does not use the perfectness of $\mathscr{A}$ except for proving that $\mathscr{A} \subset \mathscr{A}_{\alpha}$ and $\mathscr{A}_{c} \subset \mathscr{B}_{\alpha}$ for each $\alpha>0$, that is for proving a part of (II) with $\beta$ $=0$. Therefore the following definition makes sense.

Definition 5.1. A $\sigma$-ring $\mathscr{A}$ on $X$ is $\mathscr{A}_{\alpha}$-normal $(\alpha \geq 0)$ if $\mathscr{A}_{\alpha}$ is a normal ring, that is, for any two disjoint members of $\mathscr{B}_{\alpha}$ there exist disjoint members of $\mathscr{A}_{\alpha}$ containing them.

REMARK 5.2. Of course, a topological space $(X, \mathscr{A})$ is normal (respectively $F_{\sigma}$ normal) if and only if $\mathscr{A}$ is $\mathscr{A}_{0}$-normal (respectively $\mathscr{A}_{1}$-normal). As proved in [5], each perfect space is $\mathscr{A}_{1}$-normal. In fact each perfect $\sigma$-ring (in particular, each perfect space) is $\mathscr{A}_{\alpha}$-normal for any $\alpha>0$, as follows from the following observation.

PROPOSITION 5.3. Each perfect $\sigma$-ring $\mathscr{A}$ on a set $X$ is $\mathscr{A}_{\alpha}$-normal for arbitrary countable $\alpha>0$.

PROOF. [7, Theorem 2, p. 350] shows, in particular, that in a metric space $X$, every two disjoint members of $\mathscr{B}_{\alpha}$ are contained in disjoint members of $\mathscr{A}_{\alpha}(\alpha>0)$ ). (In fact, they are contained in disjoint members of $\mathscr{A}_{\alpha} \cap \mathscr{B}_{\alpha}$, but this is irrelevant for our purposes.) The argument for proving this uses merely the fact that open sets of $X$ form a perfect $\sigma$-ring.

REMARK 5.4. In addition to Remark 4.6, note that the normality case of Theorem 4.4 characterizes $\mathscr{A}_{\alpha}$-normal spaces $(\alpha \geq 0)$ too. In particular, since each perfect space $(X, \mathscr{A})$ is $\mathscr{A}_{\alpha}$-normal $(\alpha>0)$ and $\mathscr{A}_{\alpha}$-measurable functions on a perfect space are just functions of class $\alpha$, Theorem 4.4 provides properties of perfect spaces in terms of inserting and extending functions of class $\alpha$ as well as in terms of separating disjoint sets of multiplicative class $\alpha$ by functions of class $\alpha(\alpha>0)$. We state one of them explicitly. 
THEOREM 5.5. If $X$ is a perfect space and $\alpha>0$ is a countable ordinal, then every class $\alpha$ function from a subset of multiplicative class $\alpha$ to $[0,1]$ has a class $\alpha$ extension to the whole of $X$.

REMARK 5.6. The above theorem partially improves one classical result of $[7$, Theorem, p. 434], in which $X$ is a metric space and the function takes values in a complete separable metric space. Note that the proof of [7] is not valid for nonmetrizable spaces.

With $\alpha=1$, Theorem 5.5 is [5, Corollary 2.11].

REMARK 5.7. For $\mathscr{A}$ a perfect $\sigma$-ring on a set $X$, the normality case of the Insertion Theorem of Theorem 4.4 was previously known under the hypothesis that there exists a ring $\mathscr{R}$ of real-valued functions on $X$ such that each lower (respectively upper) $\mathscr{A}$-measurable function on $X$ is a pointwise limit of an increasing (respectively decreasing) sequence of functions from $\mathscr{R}$ (see [14, Chapter V, 10.1]).

\section{References}

[1] R. L. Blair, 'Extension of Lebesgue sets and of real-valued functions', Czechoslovak Math. J. 31 (1981), 63-74.

[2] R. Engelking, General topology (PWN, Warsaw, 1977).

[3] L. Gillman and M. Jerison, Rings of continuous functions (Springer, Berlin, 1976).

[4] M. Katětov, 'On real-valued functions in topological spaces', Fund. Math. 38 (1951), 85-91 'Correction', ibid. 40 (1953), 203-205.

[5] W. Kotzé, 'Functions of class one and well-behaved spaces', Rend. Mat. Appl. 4 (1984), 139-156.

[6] T. Kubiak, 'Normality versus extremal disconnectedness', preprint.

[7] K. Kuratowski, Topology, Vol. 1 (Academic Press, New York, 1966).

[8] S. S. Kutateladze, Foundations of functional analysis (Nauka, Novosibirsk, 1983) (in Russian).

[9] E. P. Lane, 'A sufficient condition for the insertion of a continuous function', Proc. Amer. Math. Soc. 49 (1975), 90-94.

[10] — 'Insertion of a continuous function', Topology Proc. 4 (1979), 463-478.

[11] S. Mrówka, 'On some approximation theorems', Nieuw Arch. Wisk. 16 (1968), 94-111.

[12] I. Mustafa, 'Insertion of Darboux Baire one function', Real Anal. Exchange 12 (1986/87), 458-467.

[13] D. Preiss and J. Vilimovsky, 'In-between theorems in uniform spaces', Trans. Amer. Math. Soc. 261 (1980), 483-501.

[14] R. Sikorski, Real functions, Vol. 1 (PNW, Warsaw, 1958) (in Polish).

[15] T. P. Speed, 'On rings of sets II. zero sets', J. Austral. Math. Soc. Ser. A 16 (1973), 185-199.

[16] H. Tong, 'Some characterizations of normal and perfectly normal spaces', Duke Math.J. 19 (1952), 289-292.

Department of Mathematics

(Pure and Applied)

Rhodes University

Grahamstown 6140

South Africa
Institute of Mathematics Adam Mickiewicz University Matejki 48/49 60-769 Poznań Poland 